\title{
TRATAMIENTO DEL REFLUJO VESICOURETERAL EN NIÑOS CON MIELOMENINGOCELE
}

\author{
Ricardo González' y Barbara M. Ludwikowski².
}

Urología Pediátrica'. Al DuPont Hospital for Children. Thomas Jefferson University. Wilmington. Delaware and Philadelphia. Pennsylvania. USA.

Pediatric Surgery². Landesklinikum Salzburg. Paracelsus Medical University. Salzburg. Austria.

\begin{abstract}
Resumen.- OBJETIVO: Hacer una revisión de la literatura sobre el tópico de reflujo vesicoureteral (RVU) en niños con mielomeningocele.

MÉTODOS: Búsqueda en PubMed bajo los títulos: vesicoureteral reflux y myelomemingocele.

RESULTADOS: Entre 1981 y 2007 se encontraron 340 artículos de los cuales 61 se consideraron pertinentes y 48 se usaron como referencias para esta mononografía. Se discute la patogénesis del RVU en la vejiga neurógena, la efectividad del tratamiento conservador con cateterismo intermitente y anticolinérgicos, así como la indicación y efectividad de diversos procedimientos quirúrgicos incluyendo el reimplante ureteral, la inyección subureteral de varios agentes, la ampliación vesical, vesicostomía cutánea, dilatación uretral, transuretero-ureterostomía e inyección de toxina botulínica.
\end{abstract}

CONCLUSIÓN: EI RVU juega un papel importante en el desarrollo de daño renal en infantes y niños nacidos con mielomeningocele. La nefropatía puede ser prevenida con una evaluación y seguimiento adecuados y con intervención terapéutica oportuna. La insuficiencia renal en estos pacientes siempre refleja el fracaso de la atención médica o de las condiciones sociales o socio-económicas.

Palabras clave: Reflujo vesicoureteral. Mieloneningocele. Niños.

Summary.- OBJECTIVES: To perform a bibliographic review on the topic of vesicoureteral reflux (VUR) in children with myelomeningocele.

METHODS: PubMed search using the terms: vesicoureteral reflux and myelomeningocele.

RESULTS: Between 1981 and 2007340 articles were found, 61 of which were considered adequate, and 48 were used as references for this monographic issue. We discuss the pathogenesis of VUR in neurogenic bladder, the efficacy of conservative treatment with clean intermittent catheterization and anticholinergic drugs, as well as the indications and efficacy of various surgical procedures including ureteral reimplantation, subureteral injection of various agents, bladder augmentation, cutaneous vesicostomy, urethral dilation, transureteral-ureterostomy and botulin toxin injection.

CONCLUSIONS: Vesicoureteral reflux plays an important role in the development of renal damage in infants and children with congenital myelomeningocele. Nephropathy may be prevented with adequate evaluation and follow-up and timely therapeutic intervention. Renal failure in these patients always reflects the failure of medical care or social/socioeconomic conditions.

Keywords: Vesicoureteral reflux. Myelomeningocele. Children. 


\section{INTRODUCCIÓN}

El RVU en niños con vejiga neurógena es un fenómeno secundario que con frecuencia causa daño renal y que sin tratamiento puede llevar a la insuficiencia renal crónica. Aunque es difícil encontrar documentación publicada, en la primera mitad del siglo XX, la insuficiencia renal era una causa importante de morbilidad y mortalidad en niños con espina bífida. En 1967, Eckstein comunica que la insuficiencia renal fue la causa de muerte en el $8 \%$ de niños con mielomeningocele (1). En 1982, Woods y Atwell reportan una mortalidad por causas diversas del $30 \%$ en el primer año de vida (2). En contraste con esta información, en la actualidad en Estados Unidos y Europa occidental el fallo renal en niños con espina bífida es excepcionalmente raro. Entre 280 adultos con espina bífida seguidos en una clínica en Belfast durante 10 años, hubo 18 muertes $(6.4 \%)$ de las cuales 4 fueron por insuficiencia renal (3). Existe también en la literatura un puñado de comunicaciones acerca del transplante renal o diálisis en niños con espina bífida o agenesia del sacro (4-7). Este cambio se debe principalmente al reconocimiento del papel preponderante de la disfunción vesical en el desarrollo del daño renal así como de la adopción de medidas para prevenirlo desde una temprana edad (8). En esta monografía discutiremos la evaluación y tratamiento urológico precoces de la disfunción vesical y RVU con miras a prevenir el daño renal.

\section{INCIDENCIA}

En el curso de la vida, más de la mitad de los niños con mielomeningocele tendrán $\operatorname{RVU}(2,9)$. En comunicaciones más recientes de series en las cuales el tratamiento con cateterismo intermitente, anticolinérgicos y agentes antibacterianos se inició en una etapa temprana de la vida, la incidencia de reflujo se ha visto reducida al $19 \%$ con cicatrices renales en solamente 6 de 27 pacientes con RVU (8).

\section{PATOGÉNESIS}

Aunque en algunos niños el mielomeningocele puede coexistir con RVU primario, en la mayoría el RVU se desarrolla a consecuencia de la disfunción vesical. En un estudio publicado por Sidi y cols., se investigó una serie de neonatos en forma prospectiva. Los pacientes fueron divididos en 2 grupos, uno de alto otro de bajo riesgo de desarrollar alteraciones de las vías urinarias altas. Se consideraron de alto riesgo a los niños con disinergia vesico-esfinteriana o vejigas de baja acomodación. Estos pacientes representaron el $30 \%$ del total. La mayoría de estos niños presentaban alteraciones radiológicas en la evaluación inicial y mejoraron con anticolinérgicos y cateterismo intermitente. En cambio, la mayoría de los pacientes con buena acomodación vesical o esfínteres desnervados tuvieron estudios iniciales normales y permanecieron normales sin intervención (10). Éste y otros estudios así como la experiencia acumulada con pacientes con lesiones medulares traumáticas y el resultado del tratamiento actual, apoyan la validez de la siguiente hipótesis sobre la cadena de even- tos que llevan al reflujo y el daño renal. La hiperactividad del detrusor y la disminución de la acomodación vesical con un esfínter tónico causan presiones intravesicales elevadas e hipertrofia del músculo detrusor que se manifiesta radiologicamente como trabeculación vesical. Con el tiempo, esto lleva a la falla de la unión ureterovesical, el reflujo y dilatación de las vías altas. En este escenario, la infección urinaria aumenta el riesgo de cicatrices renales $(11,12)$. En niños con mielomeningocele sin RVU, la bacteriuria asintomática no causa daño renal (13). No se sabe con certeza si el RVU con presiones intravesicales elevadas es prejudicial en ausencia de infección. El neonato corre también el riesgo de desarrollar hidronefrosis, aún sin reflujo, cuando después del cierre del defecto hay retención urinaria crónica durante la etapa de shock espinal.

\section{EVALUACIÓN}

La evaluación dirigida a detectar y prevenir RVU en niños con mielomeningocele debe comenzar en el período neonatal. Tan temprano como sea posible se debe hacer una ecografía renal y vesical. La primera cistografía miccional deberá hacerse cuando el recién nacido pueda ser movilizado. Si se cuenta con la posibilidad de realizar estudios video-urodinámicos, se podrá también hacer la primera cistomanometría en este momento. Debemos tener siempre presente que la function vesical de estos niños cambia durante su crecimiento, los estudios deben ser repetidos a través de la infancia, niñez y adolescencia. Por ejemplo, es común que la vejiga sea inicialment atónica par luego convertirse en una de alta presión. Los estudios urodinámicos pueden señalar factores de riesgo, a saber: 1) vejiga trabeculada, 2) RVU, 3) capacidad vesical disminuída, 4) acomodación vesical disminuída, 5) vejiga hiperactiva, 6) disinergia vesico-esfinteriana. La disinergia puede diagnosticarse en forma directa realizando una cistomanometría simultánea con un electromiograma con agujas insertadas en la zona del esfínter externo. Signos indirectos de disinergia incluyen la dilatación de la uretra proximal durante la contracción vesical, o la presencia de trabeculación vesical. Aunque muchos consideran la presión de pérdida elevada como un signo indirecto de resistencia uretral elevada, el valor de este parámetro en lactantes y niños no ha sido establecido. Repetimos los estudios urodinámicos cada 4 a 6 meses en el primer año de vida y luego anualmente.

\section{TRATAMIENTO CLÍNICO}

Los elementos centrales del tratamiento del RVU asociado a la vejiga neurógena son 1) profilaxis antibacteriana, 2) cateterismo intermitente. 3) agentes anticolinérgicos. Estas medidas, cuando se aplican a pacientes clasificados urodinámicamente como de alto riesgo, también pueden prevenir el desarrollo del RVU (8). Kasabian y cols. trataron 21 niños con alto riesgo para desarrollar RVU, con cateterismo intermitente y oxibutinina y lo previnieron en todos menos 1 caso (14). También parece aceptable esperar hasta que haya RVU para iniciar el tratamiento siempre y cuando los pacientes sean controlados frecuentemente y el tratamiento se comience en forma temprana. 
Kass and Koff fueron de los primeros en publicar el uso de cateterismo intermitente para tratar el RVU de causa neurógena (15). Ellos recomendaron el tratamiento con cateterismo intermitente y agentes antibacterianos y demostraron que en muchos casos de RVU de grado bajo el reflujo desaparecía. Ellos reservaron el tratamiento quirúrgico para los pacientes con bacteriruria recurrente o infecciones febriles. La resolución del RVU de grado alto fue excepcional. Sidi y cols. publicaron resultados del seguimiento de RVU en 30 niños con mielomeningocele por un promedio de 5 años. El RVU cesó en 6 de 16 casos de grado bajo con solamente agentes antibacterianos y observación. En 6 casos adicionales, el RVU de grado bajo se resolvió con cateterismo intermitente y anticolinérgicos y solamente 2 de los 16 pacientes requirieron intervención quirúrgica. Por otra parte, 14 de los 16 pacientes con RVU de alto grado necesitaron cirugía (16). Estos resultados han sido duplicados en otros estudios. Por ejemplo, en una serie de 166 pacientes se encontraron 48 casos de RVU de los cuales 21 se resolvieron sin cirugía (17).

Creemos que en niños con RVU, conviene usar un catéter estéril para cada cateterismo, evitando reusarlos. Sin embargo no hay publicaciones que apoyen esta recomendación (18). La oxibutinina ha sido usada sin problemas incluso en neonatos y lactantes (19). Puede ser administrada por vía oral $10,2 \mathrm{mg} / \mathrm{kg} /$ día dividida en 3 dosis) La respuesta al tratamiento debe ser controlada urodinámicamente. La dosis puede ser también instilada en la vejiga $(0.1 \mathrm{mg} / \mathrm{kg}$ dos veces por día) (20). En algunos países se consiguen soluciones estériles del fármaco. Como agente antibacteriano preferimos la trimetoprima (1-2 mg/ $\mathrm{kg} /$ día). Algunos prefieren el uso de cefalosporinas en las primeras 6 semanas de vida(10 mg/ $\mathrm{kg} /$ día). Después de los 3 meses de edad, puede también usarse la nitrofurantoína (1-2 mg/kg/día) o la trimetoprima combinada con sulfimetoxazol. Schlager reportó que la nitrofurantoína disminuye el número de infecciones febriles causadas por $E$. coli en niños con mielomeningocele pero no cambia la alta incidencia de bacteriuria en estos casos (21). El uso profiláctico de nitrofurantoína lleva a reemplazar la flora vesical de E.coli por Klebsiella y Pseudomonas. También usada en muchos lugares en las primeras semanas de vida es la amoxicilina. Su uso, sin embargo, conlleva el riesgo del desarrollo de E.coli multiresistente. A pesar de la falta de estudios controlados, creemos que el uso de terapia antibacteriana profiláctica es beneficioso para los niños con RVU y mielomeningocele.

\section{INTERVENCIONES QUIRÚRGICAS}

Entre las intervenciones usadas para el tratamiento quirúrgico del RVU contamos con la vesicostomía cutánea, el reimplante ureteral, la inyección subureteral de substancias que alteran la unión vesicoureteral, la dilatación uretral y la inyección de toxina botulínica en el detrusor. El tratamiento quirúrgico nunca debe ser el tratamiento inicial en estos casos. En todos los casos el tratamiento inicial debe ser el cateterismo intermitente, anticolinérgicos y agentes antibacterianos. Reservamos la intervención quirúrgica para aquellos pacientes en los cuales se desarrollan infecciones febriles, nuevas cicatrices renales o reflujo de grado alto persistente. También debe contemplarse la cirugía antireflujo en los pacientes con RVU que se someten a operaciones para corregir la incontinencia urinaria.

Vesicostomía cutánea. Aplicamos este procedimiento a lactantes y neonatos en los cuales, por razones anatómicas o familiares, no puede efectuarse el cateterismo intermitente o no toleran los anticolinérgicos (22-24).

Reimplante ureteral. El reimplante ureteral puede llevarse a cabo de forma segura y efectiva en niños con vejiga neurogénica en los cuales, la acomodación vesical es adecuada y la vejiga se vacía regularmente por medio del cateterismo $(9,15,23)$. Pare el reimplante ureteral preferimos la técnica de Cohen. La persistencia del reflujo es rara después de la operación, pero probablemente el riesgo de obstrucción es algo mayor que en pacientes con vejigas normales $(9,25,26)$. El reimplante ureteral no compromete la acomodación vesical cuando esta era adecuada antes de la operación (27).

Inyección subureteral. La inyección endoscópica de substancias que alteran la unión vesicoureteral con miras a curar el reflujo ha sido empleada más de 20 años como una alternativa al reimplante tanto para el RVU primario como para el secundario a la disfunción vesical neurogénica. Las substancias usadas incluyen la pasta de politetrafluoroetilene, el colágeno bovino, el polidimetilsiloxane y más recientemente dextranomer/ ácido hialurónico. A pesar de que esta última sustancia es la más usada en la actualidad, no hay publicaciones que analicen el resultado de la inyección de dextranomer/ ácido hialurónico en niños con mielomeningocele. En una serie de pacientes con RVU complejo publicada por Perez Brayfield y cols., que incluyó 11 niños con vejiga neurogénica, el éxito de la primera inyección fue del 68 \% (28). El colágeno ha sido prácticamente abandonado debido al poco éxito y la alta incidencia de recidivas $(29,30)$. La mayoría de las series publicadas analizaron el resultado de la inyección de pasta de politetrafluoroetilene, una sustancia no aprobada para este uso en muchos países. El éxito del tratamiento varió desde menos del 50 hasta más del $70 \%(26,31-33)$. Resultados comparables han sido reportados con el uso del polidimetilsiloxane (34). Así como es el caso con el reimplante vesicoureteral, existe un pequeño riesgo de obstrucción ureteral después del uso de agentes inyectables (35).

Ampliación vesical. La cirugía para ampliar la vejiga está indicada para el tratamiento de la capacidad y acomodación disminuidas que no responden al tratamiento farmacológico. El objetivo de esta cirugía es crear un reservorio para almacenar la orina a baja presión para proteger los riñones y mejorar la continencia. Cuando existe RVU asociado, los ureteres pueden ser reimplantados en el momento de la cistoplastia con buenos resultados (36). Sin embargo, el reimplante puede no ser necesario en muchos casos. En 1991, Nasrallah y Aliabadi observaron resolución del RVU en 12 de 14 casos de niños con vejiga neurogénica sometidos a ampliación sin reimplante (37). En una serie de más pacientes en Teheran, se observó resolución del RVU en $85 \%$ de los pacientes con ampliación sin reimplante (38). López Pereira y cols. (39) y otros, co- 
municaron resultados similares (40). Aunque la mayoría de las comunicaciones se refieren a la enterocistoplastia, otros han reportado resultados similares con la miomectomía del detrusor $(8,41)$. En nuestra experiencia así como la de otros, esta operación no ha sido efectiva para ampliar la vejiga (42).

Otros procedimientos. La transuretero-ureterostomía pude ser útil en casos seleccionados de reflujo unilateral con buena capacidad y acomodación vesical (43). Este procedimiento puede llevarse a cabo con laparoscopia (44).

La inyección de toxina botulínica tipo $A$ en el detrusor ha sido usada en niños con el objetivo de mejorar la capacidad y acomodación vesical. Su seguridad y eficacia están bien establecidas (45) pero el efecto favorable tiene un duración promedio de 10 meses (46). Algunos autores recomiendan inyecciones repetidas (47) pero este tratamiento parece más un método para posponer la ampliación que un substituto a la misma. El papel de la toxina botulínica en el tratamiento del reflujo no ha sido establecido.

Finalmente, cabe mencionar la dilatación uretral como otra medida temporal para mejorar la acomodación vesical en niños con mielomeningocele (48).

\section{CONCLUSIONES}

El RVU es un factor de riesgo importante en el desarrollo del daño renal en el 50 \% niños con mielomeningocele. En general es el resultado de la existencia de condiciones desfavorables para el almacenamiento de orina a baja presión en la vejiga o a presiones elevadas durante la micción. En sus estadios iniciales, puede resolverse con medidas dirigidas a mejorar la acomodación y vaciado vesical, vale decir, cateterismo intermitente y anticolinérgicos. Como en el caso del reflujo primario, la profilaxis antibacteriana es también importante. Estas mismas medidas, pueden usarse también en forma preventiva en todos los niños con mielomeningocele para prevenir el desarrollo de reflujo y proteger los riñones.

La presencia de infecciones febriles or nuevas cicatrices renales son indicaciones para intervenir quirúrgicamente. El buen manejo vesical pre y postoperatorio son condiciones esenciales para el éxito del reimplante o la inyección subureteral. Cuando la capacidad y acomodación vesical no mejoran con anticolinérgicos, laq ampliación vesical puede de por sí eliminar el reflujo en muchos casos. Cuando el RVU persiste después de la ampliación, éste puede ser controlado endoscópicamente. La vesicostomía cutánea, la dilatación uretral y la inyección de toxina botulínica son mediadas temporarias.

El daño renal en niños nacidos con mielomeningocele es siempre prevenible con una adecuada evaluación, seguimiento e intervención oportuna. La insuficiencia renal en el paciente con mielomeningocele es siempre el resultado de una falla en el tratamiento, el origen de la cual puede ser médico, social o socioeconómico.

\section{BIBLIOGRAFÍA y LECTURAS RECOMENDADAS (*lectura de interés $y^{* *}$ lectura fundamental)}

1. ECKSTEIN, H.; COOPER, D.; HOWARD, E. y cols.: y cols.: "Cause of death in children with myelomeningocele or hydrocephalus". Archives of Diseases of Childhood, 42: 163, 1967.

2. WOODS, C.; ATWELL, J.: "Vesico-ureteric reflux in the neuropathic bladder with particular refernce to the development of renal scarring”. Eur. Urol., 8: 23, 1982.

3. McDONNELL, G.; McCANN, J.: "Why do adults with spina bifida and hydrocephalus die? A clinic based study". Eur. J. Pediat. Surg., 10: 31, 2000.

4. JACHIMIAK, B.; JARMOLINSKI, T.: "A child with myelomeningocele as a dialytic patient". Przegl. Lek., 63: 176, 2006.

5. KARI; J.: "Neuropathic bladder as a cause of chronic renal failure in children in developing countries". Pediatric Nephrology, 21: 517, 2006.

6. HAMDI, M.; MORAN, P.; LITTLE, D. y cols.: "Successful renal transplantation in children with spina bifida: Long term single center expereince". Pediatric Transplantation, 8: 167, 2004.

7. MENDIZABAL, S.; ESTORNELL, F.; ZAMORA, I. y cols.: "Renal transplantation in children with severe bladder dysfunction". J. Urol., 173: 226, 2005.

8. DIK, P.; KLIJN, A.; GOOL, J.V. y cols.: "Early start of therapy preserves kidney function in spina bifida patients". Eur. Urol., 49: 908, 2006.

9. SIDI, A.; PENG, W.; GONZÁLEZ, R.: "Vesicoureteral reflux in children with myelodysplasia". J. Urol., 1986: 329, 1986.

10. SIDI, A.; DYKSTRA, D.; GONZÁLEZ, R.: "The value of urodynamic testing in the management of neonates with myelodsyplasia: A prospective study". J. Urol., 135: 90, 1986.

11. MCLORIE G, PEREZ-MARERO P, CSIMA A, Churchill M. Determinants of hydronephrosis and renal injury in patients with myelomeningocele. J. Urol. 1988;140(5 pt 2):1289-92.

12. McGUIRE, E.; WOODSIDE, J.; BORDEN, T.: "Upper urinary tract deterioration in patients with myelodysplasia and detrusor hypertonia: A follow up study". J. Urol., 1983: 823, 1983.

13. OTTOLINI, M.; SHAER, C.; RUSHTON, H. y cols.: "Relationship of asymptomatic bacteriuria and renal scarring in children with neuropathic bladders who are practicing intermittent catheterization”. Journal of Pediatrics, 127: 368, 1995.

14. KASABIAN, N.; BAUER, S.; DYRO, F. y cols.: "The prophylactic value of clean intermittent catheterization and anticholinergic medication in newborns and infants with myelodysplasia at risk of developing urinary tract deterioration". American Journal of Diseases of Childhood, 146: 840, 1992.

15. KASS, E.; KOFF, S.: "The management of vesicoureteral reflux in children with neurogenic bladders". Zeitschrift für Kinderchirurgie, 34: 379, 1981.

16. SIDI, A.; PENG, W.; GONZÁLEZ, R.: "Vesicoureteral reflux in children with myelodysplasia: Natural history and results of treatment". Journal of Urology, 136: 329, 1986. 
17. MERLINI, E.; BESEGHI, U.; CASTRO, R.D. y cols.: "Treatment of reflux in the neurogenic bladder". BJU, 72: 969, 1993.

18. SCHLAGER, T.; CLARK, M.; ANDERSON, S.: "Effect of a sigle-use sterile catheter for each void on the frequency of bacteriuria in children with neurogenic bladder on intermittent catheterizaztion for bladder emtying”. Pediatrics, 108: 71,m 2001.

19. MIALDEA, R.L.; MARTIN-CRESPO, R.; HERNANDEZ, E. y cols.: "Use of oral anticholinergic therapy in children under 1 year of age with high risk bladder". Cirugía Pediátrica, 18: 3, 2005.

20. AMARK, P.; BUSSMAN, G.; EKSBORG, S.: "Follow up of long time treatment with intravesical oxybutynin for neurogenic bladder in children". Eur. Urol., 34: 148, 1998.

21. SCHLAGER, T.; ANDERSON, S.; TRUDELL, J. y cols.: "Nitrofurantoin prophylaxis for bacteriuria and urinary tract infection in children with neurogenic bladder on intermittent catheterization". Journal of Pediatrics, 132: 704, 1998.

22. ZARAGOZA, J.Q.; HINAREJOS, C.D.; DURBA, A.S y cols.: "Vesicostomy in children. Our experience with 43 patients". Actas Urol. Esp., 27: 33, 2003.

23. AGARWAL, S.; KHOURY, A.; ABRAHAMSON, R. y cols.: "Outcome analysis of vesicoureteral reflux in children with myelodysplasia”. J. Urol., 157: 980, 1997.

24. MORRISROE, S.; O'CONNOR, R.; NANIGIAN, D. y cols.: "Vesicostomy revisited: The best treatment for the hostile bladder in myelodysplastic children". BJU International, 96: 397, 2005.

25. KAPLAN, W.; FIRLIT, C.: "Management of reflux in the myelodysplastic child". J. Urol., 129: 1195, 1983.

26. GRANATA, C.; BUFFA, P.; ROSAVENDA, E.D. y cols.: "Treatment of vesico-ureteric reflux in children with neuropathic bladder: A comparison of surgical and endoscopic correction”. J. of Pediat. Surg., 34: 1836, 1999.

27. GOTOH, M.; KONDO, A.; MIYAKE, K.: "Bladder compliance in myelodysplastic children: Does antireflux surgery compromise it?". Urologia Internationalis, 47: 63, 1991.

28. PEREZ-BRAYFIELD, M.; KIRSCH, A.; HENSLE, T. y cols.: "Endoscopic treatment with dextranomer/hyaluronic acid for complex cases of vesicoureteral reflux". J. Urol., 172: 1614, 204.

29. HEFERKAMP, A.; MOHRING, K.; STAEHLER, G. y cols.: "Long-term efficacy of subureteral collagen injection for endoscopic treatment of vesicoureteral reflux in neurogenic bladder cases". J. Urol., 2000: 274, 2000

30. YOKOYAMA, O.; ISHIURA, Y.; SETO, C. y cols.: "Endoscopic treatment of vesicoureteral reflux in patients with myelodysplasia”. J. Urol., 155: 1882, 1996.

31. KAMINETSKY, J.; HANNA, M.: "Endoscopic treatment of vesicoureteral reflux in children with neurogenic bladders". Urology, 37: 244, 1991.

32. DEWAN, P.; GUINEY, E.: "Endoscopic correction of vesicoureteral reflux in children with spina bifida". BJU, 65: 646, 1990.

33. ENGEL, J.; PALMER, L.; CHENG, E. y cols.: "Surgical versus endoscopic correction of vesicoureteral re- flux in children with neurogenic bladder dysfunction". J. Urol., 157: 2291, 1997.

34. DURBA, A.S.; PEREZ, L.G.; MORAGUES, F.E. y cols.: "Efficacy of endoscopic treatment of scondary vesicoureteral reflux with polydimethylsiloxane". Actas Urol. Esp., 30: 692, 2006.

35. VANDERSTEEN, D.; ROUTH, J.; KIRSCH, A. y cols.: "Postoperative ureteral obstruction after subureteral injection of dextranomer/hyaluronic acid copolymer". J. Urol., 176: 1593, 2006.

36. HAYASHI, H.; YAMATAKA, A.; KANEYAMA, K. y cols.: "Review of 86 patients with myelodysplasia and neurogenic baldder who underwent sigmoidocolocystoplasty and were followed for more than 10 years". J. Urol., 176: 1806, 2006.

37. NASRALLAH, P.; ALIABADI, H.: "Bladder augmentation in patients with neurogenic bladder and vesicoureteral reflux”. J. Urol., 146: 563, 1991.

38. SIMFOROOSH, N.; TABIBI, A.; BASIRI, A. y cols.: "Is ureteral reimplantation necessary during augmentation cystoplasty in pateints with neurogenic bladder and vesicoureteral reflux?". J. Urol., 168: 1439, 2002.

39. PEREIRA, P.L.; URRUTIA, M.M.; ROMERA, R.L. y cols.: "Should we treat vesicoureteral reflux in patients who simultanoeusly under go bladder augmentation for neuropathic bladder?”. J. Urol., 165: 2259, 2001.

40. SOYLET, Y.; EMIR, H.; ILCE, Z. y cols.: "Quo vadis? Ureteric reimplantation or ignoring reflux during augmentation cystoplasty". BJU International, 94: 379, 2004.

41. RAWASHDEH, Y.; JORGENSEN, T.; OLSEN, L. y cols.: "The outcome of detrusor myotomy in children with neurogenic bladder dysfunction". J. Urol., 171: 2654, 2004.

42. MARTE, A.; DIMEGLIO, D.; CORTRUFO, A. y cols.: "A long-term follow-up of autoaugmentation in myelodysplastic children". BJU International, 82: 928, 2002.

43. PESCE, C.; COSTA, L.; CAMPOBASSO, P. y cols.: "Successful use of transureteroureterostomy in children: A clinical study". Eur. J. Pediat. Surg., 11: 395, 2001.

44. PIAGGIO, L.; GONZÁLEZ, R.: "Laparoscopic transuretero-ureterostomy. A novel approach". J. Urol., 177:2311, 2007.

45. SCHULTE-BAUKLOH, H.; MICHAEL, T.; STURZEBECHER, B, y cols.: "Botulinim-a toxin detrusor injection as a novel approach in the treatment of bladder spasticity in children with neurogenic bladder". Eur. Urol., 44: 139, 2003.

46. RICCABONA, M.; KOEN, M.; SCHINDLER, M. y cols.: "Botulinum-A-toxin injection into the detrusor: A safe alternative in the treatment of children with myelomeningocele with detrusor hyperreflexia". J. Urol., 171: 845, 2004

47. ALTAWEEL, W.; JEDNACK, R.; BILODEAU, C. y cols.: "Repeated intradetrusor botulinum toxin type A in children with neurogenic bladder due to myelomeningocele". J. Urol., 175: 1102, 2006.

48. PARK, J.; McGUIRE, E.; KOO, H. y cols.: "External urethral sphincter dilatation for the management of high risk myelomeningocele: 15-year experience". J. Urol., 165: 2383, 2001. 